\title{
Time scale of NPLI: current status and future plan of improvement
}

\author{
P Banerjee, Suman Sharma and Arundhati Chaterjee
}

National Physical Laboratory, New Delhi 110012, India

Received 16 July 2008

Published 5 December 2008

Online at stacks.iop.org/Met/45/S66

\begin{abstract}
The National Physical Laboratory, India (NPLI), is responsible for maintenance and development of the Indian Standard Time (IST) and also contributes through the GPS network to the generation of UTC coordinated by BIPM. Recently NPLI has made some special efforts to improve the uncertainty of UTC(NPLI) with respect to UTC. One basic clock ensemble algorithm is implemented to make the time scale of UTC(NPLI) by combining the intercomparison data of five caesium clocks. Some initial tests have been carried out to check the validity of this algorithm. The recent UTC - UTC(NPLI) values (shown in Circular T) and the corresponding frequency offset with respect to UTC have exhibited significant improvements. This paper describes these studies and presents the analysis of these observations.
\end{abstract}

(Some figures in this article are in colour only in the electronic version)

\section{Introduction}

National Physical Laboratory, India (NPLI), establishes, maintains and continuously improves by research the national standards of measurements and realization of the units based on the International Standard System, SI. For this purpose it is essential that the national standards of physical measurement at NPLI be traceable to international standards. The laboratory periodically carries out a comparison of national standards with the corresponding standards maintained by national metrology institutes (NMIs) of other countries. NPLI is the custodian of the time and frequency standard in India. The time group of NPLI maintains the Indian Standard Time (IST) - the national time scale and its associated time and frequency dissemination services. There have been continuing efforts to improve the performance of the time scale of NPLI with respect to Coordinated Universal Time (UTC) maintained by BIPM. The goal of each national time laboratory is to keep a local representation of UTC $(k)$ (i.e. UTC(NPLI) for NPLI), in close agreement with UTC. UTC(NPLI) uses one commercially available caesium atomic clock to maintain the time scale which is linked to BIPM through the GPS network. To realize a better time scale, the main emphasis of research is on the development of algorithms to reduce noise processes in clocks and to analyse the performance of time transfer links. The aim of this paper is to introduce the current status of time scale of NPLI based on some measures adopted recently.

\section{Recent improvement of time scale of NPLI}

\subsection{Environment of clocks}

NPLI maintains a bank of five commercially available caesium atomic clocks (model HP5071A). Earlier clocks were kept in a room with a window-mounted air conditioning unit. Since 2005, NPLI has provided environmentally controlled rooms for the primary standards of different parameters. Now the caesium clocks of NPLI are housed in a room whose temperature is maintained at $(23 \pm 1){ }^{\circ} \mathrm{C}$ and relative humidity at $(50 \pm 5) \%$.

\subsection{Determination of clock behaviour}

It is important to evaluate the performance of each clock absolutely and independently. In the first phase of clock data analysis the independent stability of each clock is determined so that proper weighting may be assigned to it when combining them. Characterization of the phase and frequency instabilities of clocks is of great importance in forming the ensemble time scale. The Allan deviation is normally used to quantify the uncertainties of a time and frequency reference source against which other time and frequency devices are calibrated [1]. The random frequency stability of an oscillator, in the time domain, may be estimated by several sample variances. The most commonly used measure is the two-sample standard deviation 
(also known as the Allan deviation) which is the square root of the two-sample zero dead-time variance (also designated as the Allan variance). The normal Allan or two-sample variance is defined as

$$
\sigma_{y}^{2}(\tau)=\frac{1}{2(M-1)} \sum_{t=1}^{M-1}\left[\bar{y}_{i}(t+\tau)-\bar{y}_{i}(t)\right]^{2},
$$

where $y_{i}(t)$ is the $i$ th of the $M$ fractional frequency values averaged over the measurement interval $\tau$.

In terms of phase data, the Allan variance may be calculated as

$\sigma_{y}^{2}(\tau)=\frac{1}{2(N-2) \tau^{2}} \sum_{t=1}^{N-2}\left[x_{i}(t+2 \tau)-2 x_{i}(t+\tau)+x_{i}(\tau)\right]^{2}$,

where $x_{i}(t)$ is the $i$ th of the $N=M+1$ phase values spaced by the measurement interval $\tau$.

The terms $x(t+\tau)$ and $x(t)$ are proportional to instantaneous time differences obtained from the comparison between two clocks at times $(t+\tau)$ and $t$. It is important to note that in any frequency and phase measurement, each clock is compared with respect to a reference clock. The measurement is therefore a relative one, and so also is the Allan deviation. Absolute Allan deviation may be directly determined [2] by intercomparison of a minimum of three clocks, assuming that the noise in all clocks is fully uncorrelated. The measured data may be assumed to correspond to $x_{j k}(t)$, or phase measurements of one clock $j$ with respect to the reference clock $k$. With the help of intercompared data one may thus find out $\sigma_{j k}(\tau)$ (the value of $\sigma_{y}(\tau)$ for clock $j$ with respect to that of clock $k$ ) using equation (2). These values of Allan deviation are, again, relative ones, not absolute ones. We may assume that the noise in all the caesium clocks is uncorrelated. So one may write $[3,4]$

$$
\sigma_{j k}^{2}(\tau)=\sigma_{j}^{2}(\tau)+\sigma_{k}^{2}(\tau) \quad j, k=1,2,3, \ldots
$$

(e.g. $\sigma_{j}(\tau)$ is the absolute value of $\sigma_{y}(\tau)$ for clock $\# j$ ).

The above equation may be used to determine the independent (absolute) value of the Allan deviation for each of the clocks from intercomparisons of several clocks. Equation (3) dictates that this method requires a minimum of three clocks to find a unique solution for $\sigma_{j}(\tau)$. Thus for three clocks, using all combinations of equation (3), it is possible to determine the Allan deviation of each clock independently by using the following relations:

$$
\begin{aligned}
& \sigma_{1}^{2}(\tau)=\frac{1}{2}\left(\left(\sigma_{12}^{2}(\tau)+\sigma_{13}^{2}(\tau)-\sigma_{23}^{2}(\tau)\right),\right. \\
& \sigma_{2}^{2}(\tau)=\frac{1}{2}\left(\left(\sigma_{23}^{2}(\tau)+\sigma_{21}^{2}(\tau)-\sigma_{13}^{2}(\tau)\right),\right. \\
& \sigma_{3}^{2}(\tau)=\frac{1}{2}\left(\left(\sigma_{32}^{2}(\tau)+\sigma_{31}^{2}(\tau)-\sigma_{12}^{2}(\tau)\right) .\right.
\end{aligned}
$$

Phase differences between pairs of clocks are measured at a regular time interval $(\tau)$. At present the intercomparison data are taken manually. This process results in gaps in the

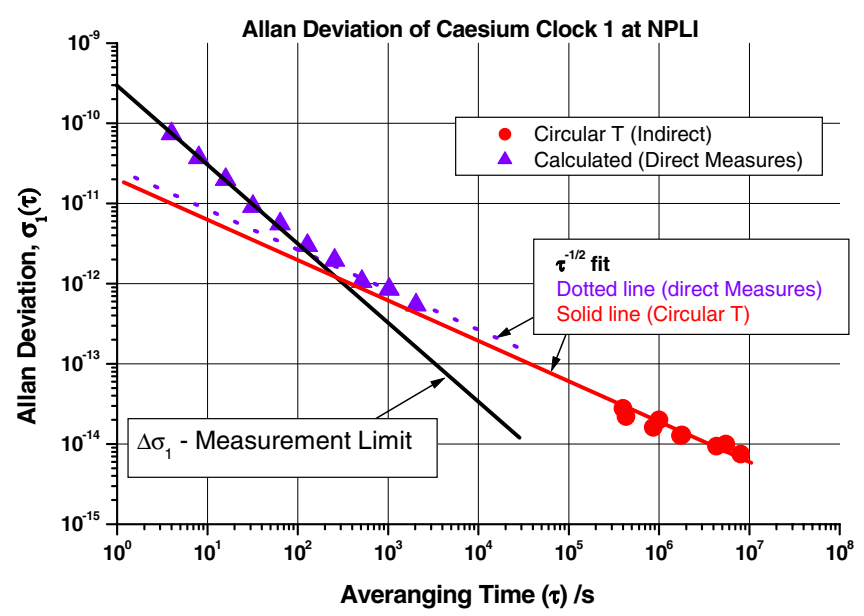

Figure 1. Determination of absolute values of Allan deviation of caesium clocks at NPLI for lower averaging time. Resolution of TIC limits the measurement capabilities.

data sets, which are filled by extrapolating from the available data. Data recording at regular intervals will be automated to provide continuous data sets, and an automatic system to take these measurements is under development. NPLI has utilized three caesium clocks to determine the absolute value of the Allan deviation of each clock for this purpose. A high resolution time interval counter (TIC) has been used to compare the phase differences between each pair of clocks. Each of the three sets of measurements has been carried out for $6 \mathrm{~h}$ continuously at the sampling rate of $4 \mathrm{~s}$. So, the minimum value of $\tau$ (i.e. averaging time) was $4 \mathrm{~s}$. These sets of measured values (i.e. $x_{j k}(\tau)$ ) are used in equation (2) to find $\sigma_{12}(\tau), \sigma_{13}(\tau)$ and $\sigma_{23}(\tau)$. Using these values of $\sigma_{j k}(\tau)$ in equations (4) to (6), $\sigma_{k}$ has been determined for each of the three caesium atomic clocks. For example, with the help of equation (4), $\sigma_{1}(\tau)$ has been calculated as shown in figure 1 .

One important point to mention here is that the aboveexplained direct method may be used to find the absolute Allan deviation for lower values of $\tau[5,6]$. This does not require the linking of clocks to BIPM, and thus, one need not wait for a long period to receive sufficient data from BIPM. But the limitation of the measurement system has to be kept in mind. For example, in these measurements, the results are limited by the resolution of the TIC. The Allan deviation for averaging times more than $100 \mathrm{~s}$ may be determined directly using a TIC with an rms resolution better than $300 \mathrm{ps}$ as shown in figure 1 .

\subsection{Time scale algorithm to ensemble atomic clocks}

NPLI at present makes use of one caesium atomic clock to maintain the UTC(NPLI) time scale. But no single clock is perfect. So the combination of several caesium clocks to generate a smoother virtual clock would have a better performance than that of a single clock. The combination of clocks to generate a virtual optimal clock is a complicated process. Many countries such as the USA and Japan have already developed this type of time scale [7]. These time scales 


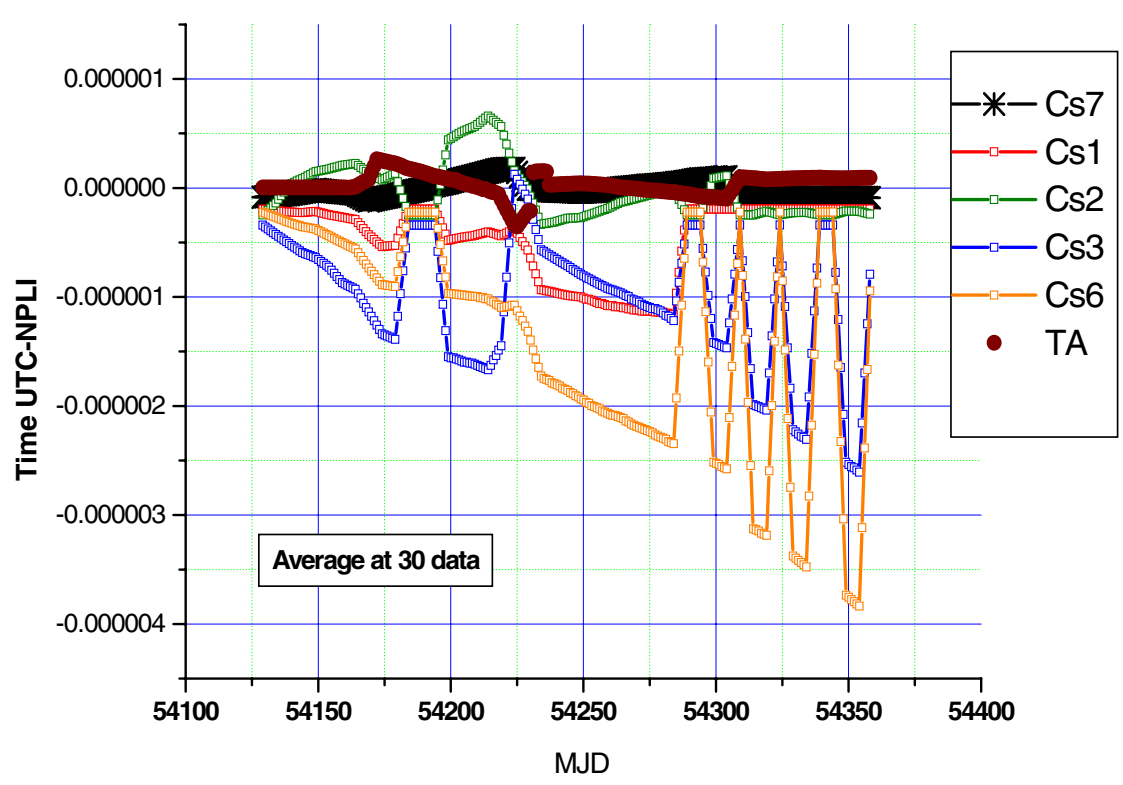

Figure 2. Typical samples of the output of the time scale algorithm.

differ widely in their implementation techniques. No package to implement a virtual time scale is available on demand or commercially. NPLI has begun to initiate such a programme with automation of the clock measurements largely based on the concept adopted in [8-10]. An initial implementation of a time scale algorithm has been tested and validated.

The most important aspect of this work is to implement a suitable algorithm for optimally combining the clocks in the ensemble based on the intercomparison data. In general, a simple average time is calculated as the sum of the available measurements divided by the total number of measurements:

$$
\mathrm{TA}(t) \equiv \sum_{i=1}^{N} \frac{1}{N} h_{i}(t)
$$

where $\operatorname{TA}(t)$ is the averaged time scale derived from $N$ clocks and $h_{i}(t)$ is the reading of clock $i$ with respect to the ideal time.

Equation (7) represents the simplest formulation of the time scale algorithm. When all the clocks are assigned equal importance without analysing their behaviour, clocks which have large instability (bad clocks) may degrade the stability of the time scale. If lower weight is assigned to bad clocks and higher weight to more stable clocks the atomic time scale becomes smoother. To introduce this scheme, equation (7) may be modified to a smoother time average as

$$
\mathrm{TA}(t) \equiv \sum_{i=1}^{N} w_{i}(t) \cdot h_{i}(t)
$$

where $w_{i}$, the weight given to clock $i$, is based on the stability of the respective clock. According to the definition of averaging, the sum of $w_{i}$ s should be unity.

Weighting is an effective tool for generating a stable time scale. The assigning of the correct weight to a particular clock behaviour must be evaluated before it is used in the time scale algorithm. The above definition has the limitations that $\mathrm{TA}(t)$ may change significantly if a clock is removed from the time scale. This limitation may be taken care of by adopting the following relation:

$$
\mathrm{TA}(t) \equiv \sum_{i=1}^{N} w_{i}(t)\left\{h_{i}(t)-\hat{x}_{i}(t)\right\}
$$

Here $\hat{x}_{i}$ is the predicted value of clock $i$ based on its performance up to last available measurements at time $t_{k} . h_{i}(t)$ cannot be obtained numerically. TA $(t)$ approaches ideal time and $h_{i}(t)$ is the difference between ideal time and clock $i$ time. So one approximates reasonably that

$$
x_{i}(t) \equiv h_{i}(t)-\mathrm{TA}(t) .
$$

The predicted time of clock $i$ may be obtained with the help of the following relation:

$$
\hat{x}_{i}\left(t_{k}\right)=x_{i}\left(t_{k-1}\right)+y_{i}\left(t_{k}\right) \cdot\left(t_{k}-t_{k-1}\right),
$$

where $y_{i}$ is the frequency offset of clock $i$ with respect to TA. It may be noted that TA may be implemented through $x_{i}$ defined by [10]. But we may only measure $X_{i j}$ which are the phase differences between clock $i$ and clock $j$. So $X_{i j}$ is related as

$$
X_{i j}\left(t_{k}\right)=h_{i s}\left(t_{k}\right)-h_{j s}\left(t_{k}\right)=x_{i}\left(t_{k}\right)-x_{j}\left(t_{k}\right) .
$$

Thus, using (9) to (12) one may write

$$
x_{j}\left(t_{k}\right)=\sum_{i} w_{i}\left(t_{k}\right)\left\{\hat{x}_{i}\left(t_{k}\right)-X_{i j}\left(t_{k}\right)\right\}
$$

Equations (11) to (13) together form the time scale ensemble algorithm and are utilized to run the time scale. The recursive series gives the time series output $x_{i}\left(t_{k}\right)$ from each run of the algorithm. The software had to be developed accordingly to implement the algorithm. The output of the software is used in the first phase to see the improvement in the time scale. 


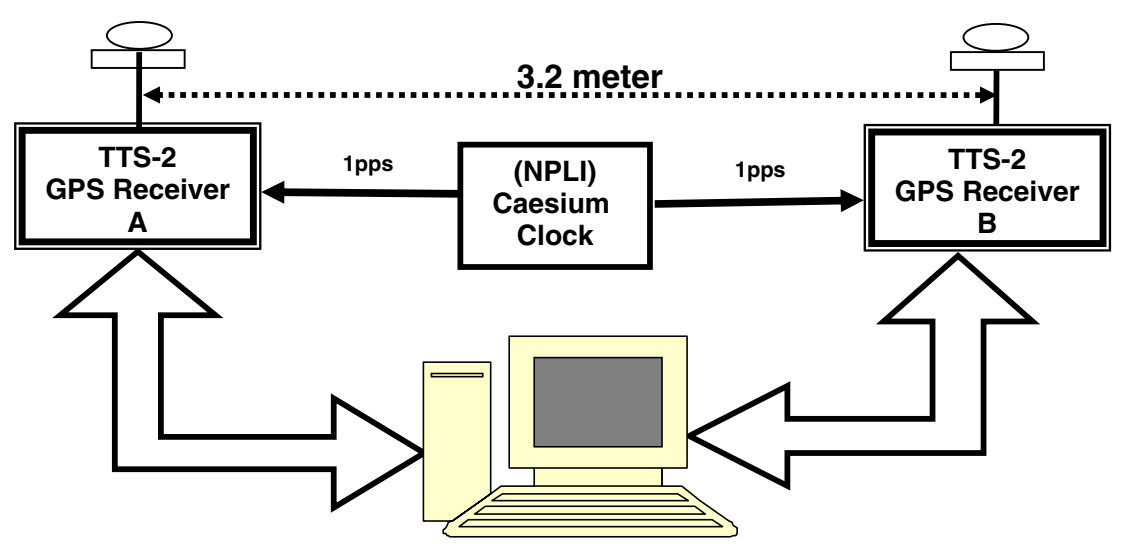

Figure 3. Experimental set-up for the special experiment campaign.

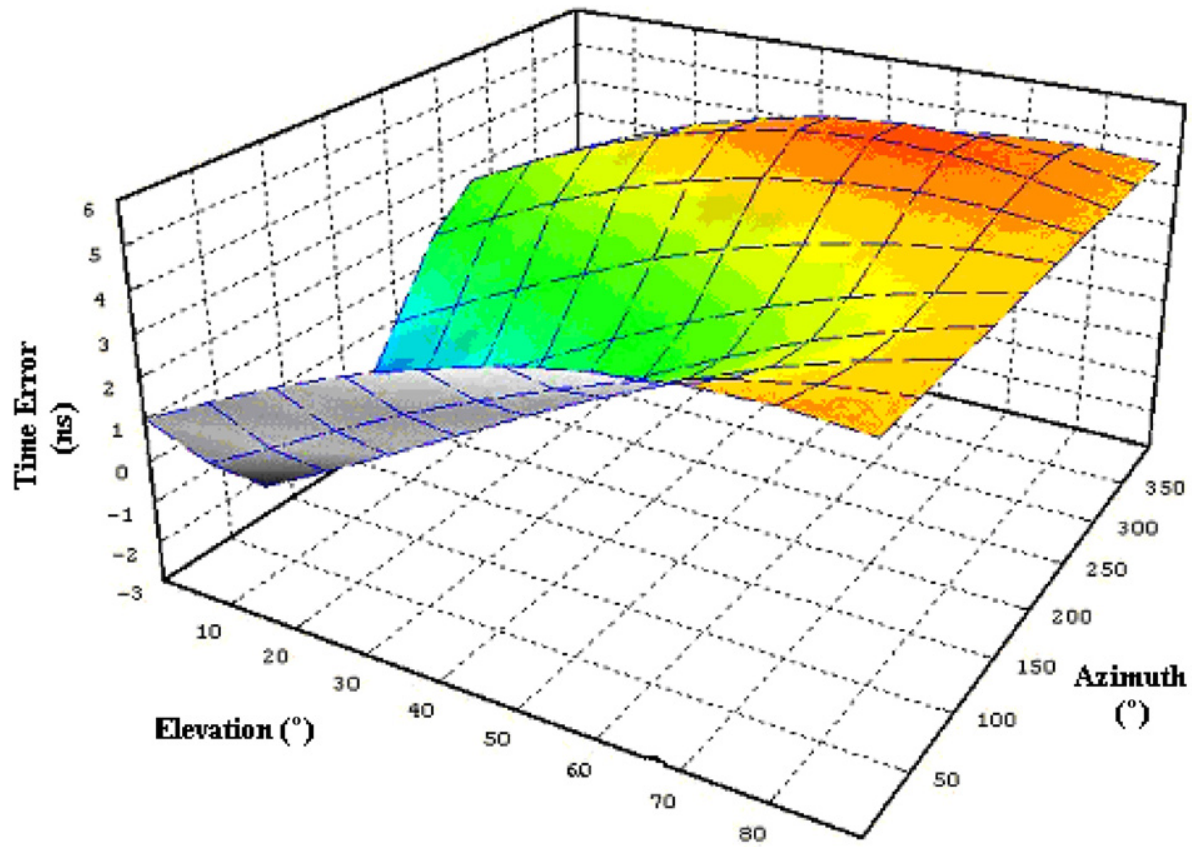

Figure 4. Effect of position error on GPS time depends on satellite elevation and azimuth with position error within a box of $100 \mathrm{~cm}$ at NPLI.

The ensemble time scale TA $(t)$, the output of this algorithm, is shown in figure 2 . The averaging time $(\tau)$ is also an important parameter in the ensemble time scale. To predict the value, the averaging time of 30 days is used. There are different techniques to determine the weight of clocks but in this TA all clocks are equally weighted for simplification. It has been observed in figure 2 that the (TA $-x_{i}$ ) ensemble time scale output is more stable than the individual clock.

\section{GPS time link with BIPM}

NPLI is already a part of BIPM's GPS network. Through this network, the time of NPLI is linked with the BIPM. The link between UTC(NPLI) and UTC is realized through a time link to Europe, namely, to PTB in Germany. After applying International GNSS Service (IGS) products of ionospheric data and precise ephemerides, the Type A uncertainty for GPS multi-channel time transfer between NPLI and Europe is reduced to $2.5 \mathrm{~ns}$. White phase noise due to the time transfer technique is observed up to an averaging time of about 1.5 days. Thus the data could be smoothed with a cut-off period of about 1.5 day. Before 2005, an Allen Osborne Associates TTR6 single channel GPS receiver was used for the time link between NPLI and BIPM. The Type B uncertainty of the link was $20 \mathrm{~ns}$. Two AOS TTS2 receivers were procured by NPLI in 2005 and were calibrated at BIPM before delivery by the manufacturer. This reduced the associated Type B uncertainty to $7 \mathrm{~ns}$. The uncertainty reported for the NPLI-PTB time link in section 6 of the BIPM Circular T is $2.5 \mathrm{~ns}$ for Type A and $7.0 \mathrm{~ns}$ [11] for Type B, giving a total uncertainty of $7.4 \mathrm{~ns}$ [12]. If the NPLI TTS2 receiver is calibrated on-site at NPLI by a travelling calibration BIPM receiver then the Type B uncertainty may be reduced further to $5.0 \mathrm{~ns}$.

It has been observed that the noise in NPLI's data is comparatively more than that in other clocks. It is necessary to identify the areas in which some corrective measures have to be taken. These identifications can only be possible through a well-planned analysis of the data. Various efforts are in progress to improve the uncertainty $U_{\mathrm{A}}$ and $U_{\mathrm{B}}$. 


\subsection{Precise coordinate determination}

(a) It has been pointed out [13] that the technique of time intercomparison demands the prior knowledge of the precise coordinates of each location. One fixed point of NPLI had been precisely determined earlier with respect to a location (IISc., Bangalore, India) which is linked to the IGS network. The same fixed point has recently been redetermined [14] with respect to another IGS station (NGRI, Hyderabad, India) for revalidation. The GPS receiver that was used for this purpose is a Bench Mark Allen Osborne Associates (AOA), 12-channel, dual frequency, geodetic Advanced Carrier Technology (ACT) receiver, with a choke ring antenna. The receiver has hyper-terminal software to download the acquired data. The data were acquired continuously for 7 days. The raw data after conversion to RINEX format were processed at NGRI using versatile GPS data processing software, Bernese Version 4.2, after obtaining the precise

(a)

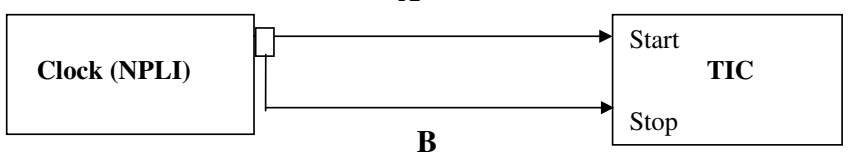

(b)

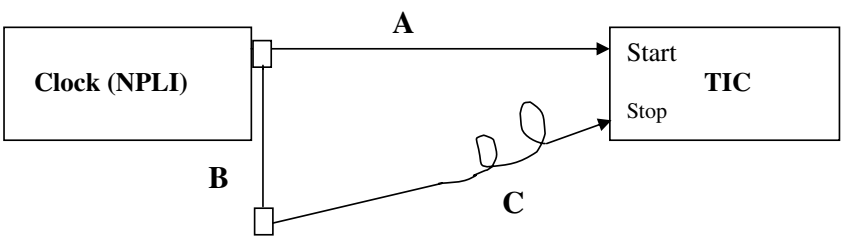

Figure 5. Calibration of the delay of cable $\mathrm{C}$ - the cable used to connect the master caesium clock and GPS receiver. orbit files from the IGS data centre. To improve the accuracy of the results, global network solutions were adopted by including many other IGS stations (COCO, HRAO, HYDE, IISc, IRKT, KIT3, LHAS, POL2, SEY1, WTZR, YAR2) in and around India. In this process, the coordinates of the fixed point of NPLI were redetermined with respect to the IGS stations with an accuracy of better than $15 \mathrm{~mm}$.

(b) The antenna may not be located precisely at the predetermined point because of practical difficulties such as the shape of the antenna structure and the need to raise the antenna height to reduce the multipath effect. An analytical expression has been derived to find the effect of the time error on the position accuracy.

In single GPS satellite mode the time error $(\Delta T$ in nanoseconds) will be directly related to the range error $\Delta R$ [15] by

$$
\begin{aligned}
\Delta T= & \frac{\Delta R}{c}=\frac{1}{30}\left[E_{x} \cdot \cos (e) \cdot \cos (a)\right. \\
& \left.+E_{y} \cdot \cos (e) \cdot \sin (a)+E_{z} \cdot \sin (e)\right],
\end{aligned}
$$

where the satellite has elevation $(e)$, azimuth $(a)$ and range $(R)$ with respect to the receiver position, $\Delta R$ is in $\mathrm{cm}$ and

$$
\begin{aligned}
E_{x}= & -\cos (L) \sin (B) \cdot \Delta x-\sin (L) \sin (B) \cdot \Delta y \\
& +\cos (B) \cdot \Delta z,
\end{aligned}
$$

where $L$ and $B$ are the nominal values of latitude and longitude of the location, respectively:

$$
\begin{aligned}
E_{y}= & -\sin (L) \cdot \Delta x+\cos (L) \cdot \Delta y, \\
E_{z}= & \cos (L) \cos (B) \cdot \Delta x+\sin (L) \cos (B) \cdot \Delta y \\
& +\sin (B) \cdot \Delta z,
\end{aligned}
$$

where $\Delta x=x-x^{\circ}, \Delta y=y-y^{\circ}, \Delta z=z-z^{\circ}$.

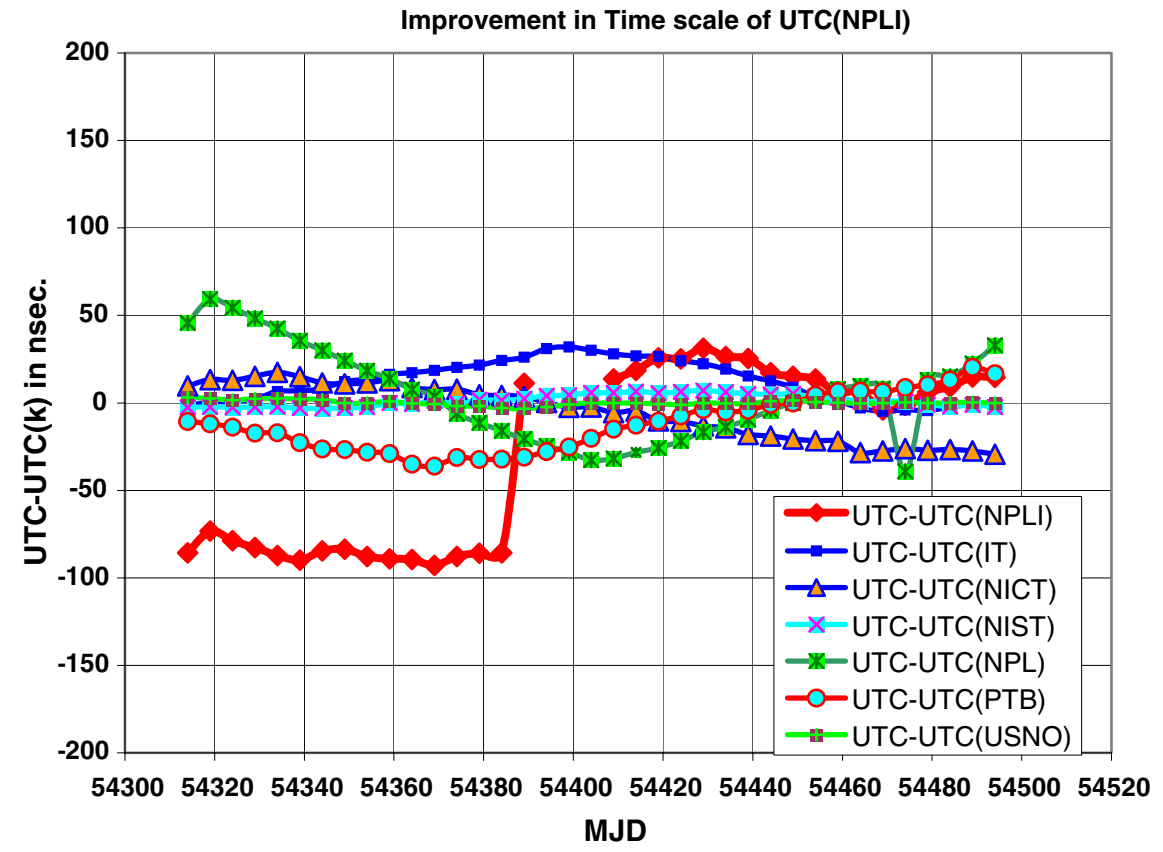

Figure 6. Improvement in UTC(NPLI) using judicious frequency adjustment is exhibited after MJD 54390. 


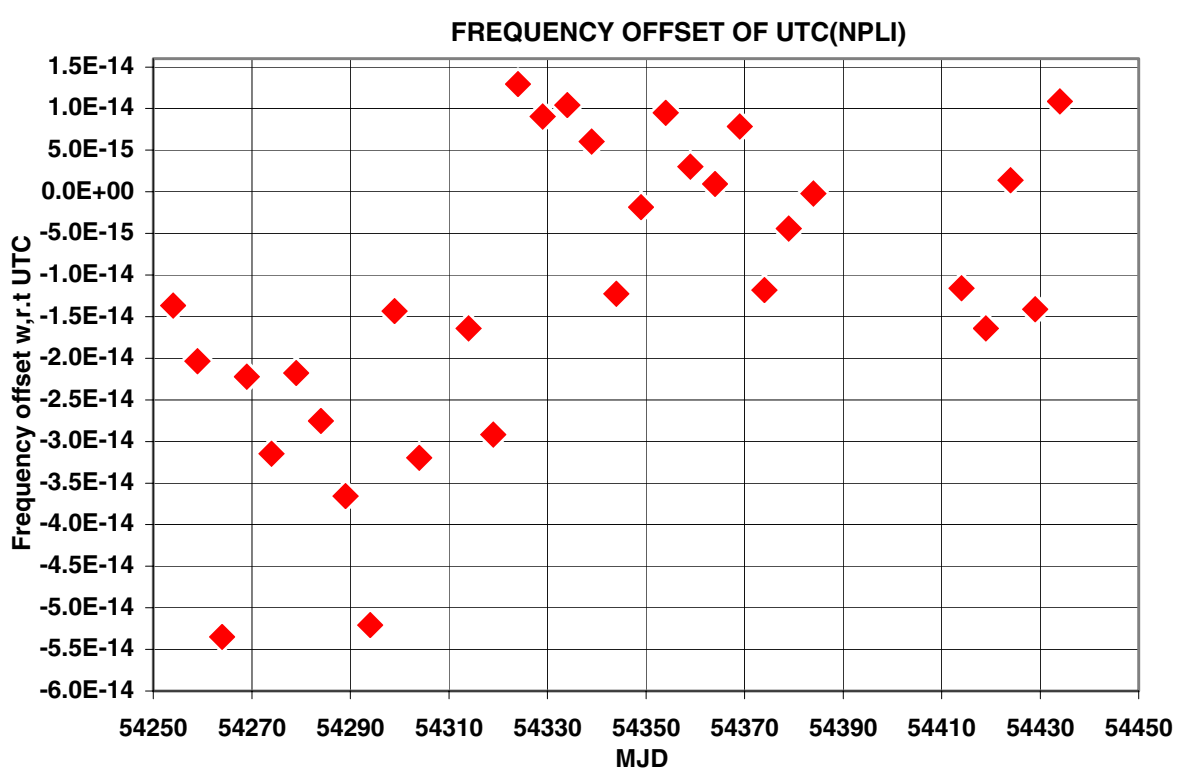

Figure 7. Status of frequency of UTC(NLPI) with respect to UTC: frequency offset fluctuates around zero within a range of $1.5 \times 10^{-14}$.

$x, y$ and $z$ are the measured values of the position in Cartesian coordinates in the horizontal system. $x^{\circ}, y^{\circ}$ and $z^{\circ}$ are the corresponding true values.

From equation (14) coupled with equations (15) to (17) the timing error $\Delta T$ may be determined. The experimental set-up related to this special campaign is shown in figure 3 and the effect of the position error on the GPS time depends on the satellite elevation and azimuth with an antenna position uncertainty of $100 \mathrm{~cm}$ in all dimensions as shown in figure 4 . This study helps in assessing the effect of the uncertainty of the position determination on the time scale.

\subsection{Calibration of antenna cable}

Keeping in mind the aim of achieving a time accuracy of few nanoseconds, it is important to measure precisely the delay of the cable used to feed $1 \mathrm{pps}$ from the reference clock to the GPS receiver. To do this, two sets of measurements have been carried out as shown in figure 5. Cable $\mathrm{C}$ is the cable whose delay is to be determined. A TIC with a resolution of $150 \mathrm{ps}$ has been used in these measurements. One may write, from figure 5 ,

$$
\begin{gathered}
\mathrm{TICa}=\text { Delay B }- \text { Delay A, } \\
\mathrm{TICb}=(\text { Delay B + Delay C })-\text { Delay A, }
\end{gathered}
$$

where TICa $=$ TIC reading for set-up at figure $8(a), \mathrm{TICb}=$ TIC reading for set-up at figure $8(b)$, Delay $\mathrm{A}=$ Delay of cable A, Delay B $=$ Delay of cable B and Delay C = Delay of cable C.

So, combining equations (18) and (19), one may write

$$
\text { Delay } \mathrm{C}=\mathrm{TICb}-\mathrm{TICa} \text {. }
$$

In the present case the delay of cable $\mathrm{C}$ has been found to be $70 \mathrm{~ns}$. This cable $\mathrm{C}$ has been used to feed $1 \mathrm{pps}$ of caesium clock to the GPS receiver.

\subsection{Multipath effect}

NPLI has two 8-channel TTS2 (developed by AOS, Poland) GPS C/A-code time receivers with the antenna separated by $3.2 \mathrm{~m}$. Ideally there should be no time difference between the GPS receivers due to the short baseline. However, we observed a significant variation in the time difference between the receivers. With a short baseline, the multipath effect is likely to be the cause of the variation. After studying the surroundings of the antennas, possible causes of multipath have been identified. There were some unused structures such as a huge dish antenna and few tall trees around the antenna which could have been sources of multipath. These structures were removed and tall branches were trimmed off from the top. These measures have reduced the rms deviation of the time difference significantly from $3.2 \mathrm{~ns}$ to $2.5 \mathrm{~ns}$.

\subsection{Noise due to error in ionospheric and tropospheric modelling}

Most of the single frequency GPS receivers utilize the Klobuchar model [16] to take care of ionospheric and tropospheric error. The efficacy of this model may not always be accurate enough to satisfy the requirement. So BIPM makes use of IGS products to improve this error. NPLI is also putting some efforts into generating better values of ionospheric and tropospheric delays with the help of in situ measurements.

\section{Current status of UTC(NPLI)}

At present, NPLI contributes to UTC by reporting GPS time comparison results and clock data to BIPM. The GPS time receiver data links UTC(NPLI) via GPS common-view time transfer to the international network of clocks from which UTC is derived. Reporting of clock data to BIPM is essential for maintaining international traceability of time and frequency in India. Over the last two years, NPLI has been working 

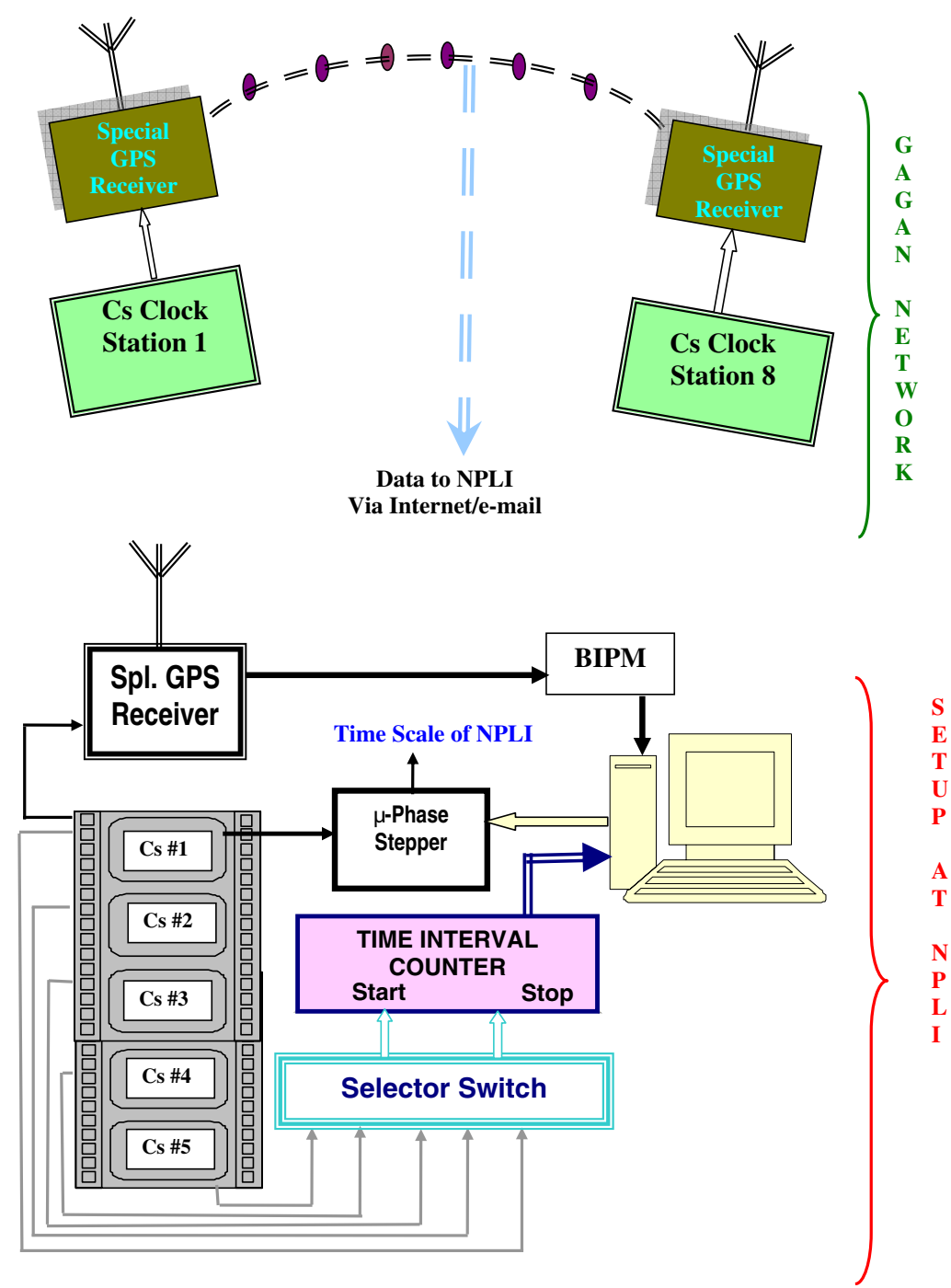

Figure 8. Schematic of the fully automated time scale system.

on improving its time keeping system and international time comparisons, and better results have been achieved as shown in figures 6 and 7 . In figure 6 one can see the improved performance of NPLI compared with some other laboratories. It is achieved by applying the frequency offset value from the algorithm to the master clock of NPLI. Figure 7 shows the frequency offset of UTC(NPLI) with respect to UTC. The improvement on the time scale after MJD 54320 is quite visible. The frequency offset after MJD 54320 varies in the range $1.3 \times 10^{-14}$ to $-1.7 \times 10^{-14}$.

Since 2005, UTC - UTC (NPLI) has been maintained to within $100 \mathrm{~ns}$, and one caesium clock contributed full weight to the TAI computation according to the BIPM Annual Report. Currently UTC - UTC(NPLI) lies well below $\pm 50 \mathrm{~ns}$. The long-term stability of UTC(NPLI) is about $6 \times 10^{-15}$ over 30 days. However, the short-term stability is about $3 \times 10^{-14}$ over 5 days.

\section{Future plan to improve UTC(NPLI)}

NPLI already has five caesium clocks and is planning to procure more in the near future. NPLI also aims to take clock intercomparison data from many caesium clocks (8 to 12 in number) of the Indian Space Research Organisation (ISRO). The corresponding plan of utilizing data from these additional clocks is shown in figure 8. By the addition of the data from these clocks to the NPLI clocks' data, NPLI may generate a more stable time scale by optimally ensembling all the available clocks' data. Data from only one caesium clock are currently submitted to the BIPM but the aim is to submit data from all five caesium atomic clocks to BIPM in the near future.

Two way satellite time and frequency transfer (TWSTFT) provides an independent technique by which a time link may be established with BIPM. TWSTFT has the capability of achieving $0.1 \mathrm{~ns}$ jitter. NPLI is planning to procure a TWSTFT system to initiate such a link. By taking some remedial measures after the study of common-view data, the uncertainty of Type A $\left(U_{\mathrm{A}}\right)$ of UTC - UTC(NPLI) has been optimized. It is now the practice to use IGS products for ephemeris and ionospheric data in order to improve the uncertainty of the time link in the GPS common-view and all-in-view methods. The accuracy of IGS products for GPS timing in the Indian subcontinent needs to be studied. The GPS link noise in the 
Time scale of NPLI: current status and future plan of improvement

NPLI system is $7.5 \mathrm{~ns}$ but we can improve this to $5 \mathrm{~ns}$ if the receiver is calibrated on-site by BIPM.

\section{Concluding remarks}

The time unit at NPLI, like that at many other NMIs, is currently facing a rapidly increasing client demand for accurate and legal time and frequency information. A TWSTFT system will join the time link system in the near future. Addition of a H-maser in the time scale is also in the pipeline. All this new equipment will make the time keeping system more reliable and give rise to improvements in the performance of UTC(NPLI). The addition of data from a number of ISRO clocks to the ensemble time algorithm will improve the stability of UTC(NPLI). NPLI is making progress to cope with the increasing demand for a precise time transfer and time keeping system.

\section{Acknowledgment}

The authors would like to thank Yuko Hanado (NICT, Japan) and Peter Whibberley (NPL, UK) for their helpful discussions and assistance in preparing this paper.

\section{References}

[1] Barnes J A et al 1971 IEEE Trans. Instrum. Meas. IM-20 105

[2] Banerjee P, Chatterjee A and Sharma S 2007 Determination of Allan deviation of cesium atomic clock for lower averaging time Indian J. Pure Appl. Phys. 45 945-9
[3] Premoli A and Tavella P 1993 A revisited three-cornered hat method for estimating frequency standard instability IEEE Trans. Instrum. Meas. 42 1-13

[4] Gray J E and Allan D W 1974 A method for estimating the frequency stability of an individual oscillator Proc. 28th Ann. Frequency Control Symp. (Atlantic City, NJ, 29-31 May) pp 243-6

[5] 2005 Annual report of the BIPM Time Section 1889

[6] Uncertainty extrapolation for T\&F CMC entries CCTF WGMRA Guideline 3 (Rev. 20021210)

[7] Tavella P and Thomas C 1991 Comparative study of time scale algorithm Metrologia 28 57-63

[8] Hanado Y et al 2003 Generating and measurement system for Japan standard time J. NICT 50 169-77

[9] Hanado Y, Imae M, Aida M, Hosokawa M, Ito H, Nakagawa F and Shimizu Y 2003 Algorithm of ensemble of atomic time J. NICT 50 157-67

[10] Hanado Y, Hosokawa M, Imamura K and Kotake N 2006 Improvement of frequency changes at Japan standard Time Electr. Eng. Japan 157 29-37

[11] Azoubib J and Lewandowski W 2002 Uncertainties of time links used for TAI Proc. 34th PTTI (Reston, VA) pp 413-24

[12] Lewandowski W, Matsakis D, Panfilo G and Tavella P 2006 The evaluation of uncertainties in [UTC $-U T C(k)$ ] Metrologia 43 278-86

[13] Langley R B 1997 The GPS error budget GPS World 8(3) 51-6

[14] Banerjee P, Chatterjee A, Verma M and Suri A K 2007 Improvements of Indian Standard Time at NPL, New Delhi Indian J. Radio Space Phys. 36 22-6

[15] Finkbleiner A T 1960 Introduction to Matrices and Linear Transformations (San Francisco and London: Freeman)

[16] Klobuchar J 1996 Ionosphere effects Global Positioning System: Theory and Applications vol I, ed B Parkinson and J Spilker (Washington, DC: AIAA) pp 485-516 chapter 12 